\title{
Yield of Computed Tomography Guided Fine Needle Aspiration in Diagnosis of Suspected Cases of Lung Cancer: Experience of a Tertiary Care Hospital of North India
}

\author{
Vipul Kumar', Rohtash Yadav ${ }^{2}$, Sushila Verma ${ }^{1}$, Ritu Aggarwal ${ }^{3}$, KB Gupta1 \\ ${ }^{1}$ Department of Respiratory Medicine \& TB, Pt BD Sharma Post Graduate Institute of Medical Sciences, Rohtak \\ (Haryana) India \\ ${ }^{2}$ Department of Radio-diagnosis, Pt BD Sharma Post Graduate Institute of Medical Sciences, Rohtak (Haryana) \\ India \\ ${ }^{3}$ Department of Microbiology, Pt BD Sharma Post Graduate Institute of Medical Sciences, Rohtak (Haryana) \\ India
}

Corresponding Author: Vipul Kumar

\begin{abstract}
Background: Lung cancer is the most commonly diagnosed malignancy across the globe. CT guided FNAC of suspicious lung masses is a widely accepted and simple diagnostic method of relatively low cost with minimal complications.
\end{abstract}

Methods: It was a prospective study conducted from February 2018 to February 2019 in PGIMS, Rohtak on thirty two patients of either sex having lung cancer suspected clinically or on Chest X-ray/CT Thorax who were subjected to computed tomography guided fine needle aspiration.

Results: Most of the patients (79.98\%) were in 41-70 years age group with $81.25 \%$ male $18.75 \%$ females and M:F 4:1. Mass was located on right side in $59 \%$ and on left side in $41 \%$ cases. Malignancy was diagnosed in $29(90.62 \%)$ cases whereas $3(9.37 \%)$ cases were inflammatory. Two patients $(6.25 \%)$ had small cell carcinoma whereas 26 patients $(65.62 \%)$ had non-small cell carcinoma. Out of these 26 patients, 14 had squamous cell carcinoma, 9 had adenocarcinoma and 3 had large cell carcinoma. One patient was found to have small round cell tumor. 3 patients $(9.37 \%)$ had pneumothorax as a complication out of which two required chest tube drainage.

Conclusions: CT guided FNAC is a simple, safe, and reliable procedure with high diagnostic accuracy for the diagnosis and sub typing of lung cancer. Though complications are rare, pneumothorax and hemoptysis are occasionally encountered but resolve with active management.

Keywords: Lung Cancer; FNAC; CT Guided; Pneumothorax

\section{INTRODUCTION}

Lung cancer is the most commonly diagnosed malignancy across the globe, especially in males. It is the leading cause of cancer related deaths with an average 5 year survival rate of $17.7 \% .^{[1]}$ Lung cancer is usually suspected on the basis of an abnormal radiographic imaging study, often in conjunction with symptoms caused by either local or systemic effects of the tumor. The modality selected to diagnose a suspected lung cancer is based on the size and location of the primary tumor in the lung, the presence of potential metastatic spread and the anticipated treatment plan. Imaging techniques do not always distinguish between malignant and benign lesions morphologically. A confirmed tissue diagnosis is essential for both treatment and staging of cancers. ${ }^{[2]}$ Early and accurate diagnosis is the key for the optimal treatment of lung cancer patients. New treatment strategies are becoming more complex, with certain novel therapeutics 
being restricted to specific histological or molecular subtypes of lung cancer, thus requiring more precise classification and performance of molecular testing such as epidermal growth factor receptor mutations. $[3,4]$

Bronchoscopy is useful to obtain biopsy sample in centrally situated lesions while computed tomographic guided transthoracic procedure is useful in peripherally located lesions. ${ }^{[5]}$ Flexible bronchoscopy has high sensitivity for the diagnosis of central lesions but low sensitivity for the peripheral lesions. ${ }^{[6]}$

FNAC was first used by Martin \& Ellis as a diagnostic tool. ${ }^{[7]}$ In 1976 Haaga \& Alfidi reported CT guided biopsy \& since then this procedure has been shown to be both effective $\&$ accurate. The diagnostic accuracy is reported to be more than $80 \%$ in benign disease \& more than $90 \%$ in malignant disease. ${ }^{[8]}$ Guided fine-needle aspiration cytology (FNAC) is often an effective and safe way to obtain the diagnostic material of lesions located in lung. Due to its high diagnostic accuracy rate along with simpler methods to treat its complications like pneumothorax, it has gained popularity among physicians, radiologists and pathologists. ${ }^{[9]}$

CT scan offers exquisite anatomical display of thoracic structures and allows percutaneous access. Intravenous contrast medium injection contributes to precise delineation of a lesion with regard to the anatomical environment. The ability to localize the needle tip consistently and accurately in the axial plane is a distinct advantage of CT over fluoroscopy or ultrasonography. There is significant versatility in selecting the entry point for FNAC because of the completeness of the CT image. All organs are visualized so that a variety of needle pathways can be chosen. It is possible to choose appropriate pathways to avoid specific organs and vascular structures.

FNAC of suspicious lung masses is a widely accepted and simple diagnostic method of relatively low cost. In patients with lung cancer that is inoperable owing to local factors or the patient's general condition, FNAC confirms the diagnosis and reveals the tumor type.

The present study was conducted to evaluate the yield of computed tomography guided fine needle aspiration cytology in diagnosis of suspected cases of lung cancer regarding its efficacy and safety profile.

\section{MATERIAL AND METHODS}

The study was conducted in Department of Respiratory Medicine and Department of Radio-diagnosis at $\mathrm{Pt}$ BD Sharma PGIMS, Rohtak. Thirty two patients of either sex having lung cancer suspected clinically or on Chest X-ray/CT Thorax were subjected to computed tomography guided fine needle aspiration after obtaining ethical approval from Institutional Ethics Committee.

Any patient suspected to have a new or enlarging solitary nodule or mass on chest radiograph, multiple nodules in patient not known to have malignancy or who had prolonged remission, hilar mass following negative bronchoscopy, Lung mass not safely approachable by ultrasonography was included in study. The exclusion criteria were uncooperative patients, patients unable to hold breath, patients with pulmonary hypertension, arterio-venous malformation, bleeding disorders, severe obstructive pulmonary disease, cardiac insufficiency, highly vascular lesion, suspected hydatid cyst and contralateral pneumonectomy.

Detailed history and clinical examination was done before performing the FNAC. Informed and written consent was taken from the patient explaining the risk and benefits of the procedure. Coagulation indices, platelet count and Platelet count APPT ratio was done. Oral anticoagulants were stopped 4 days before procedure. Six to eight hours fasting was advised to all patients; however, necessary medications such as antihypertensive drugs were given 5-6 h earlier with small amount of water. A pre procedure axial CT scan of chest was performed to localize the lesion 
accurately and to decide the best approach to do FNAC depending on the shortest distance from the lesion to the visceral surface of the lungs, except where there was presence of overlying skeletal structures or large pulmonary vessels. FNAC was performed by clinician in the presence of a pathologist and radiologist. All relevant equipments to deal with any complication were made available in the procedure room. The procedure was done by using 20 -gauge Chiba needle on Whole body spiral CT scanner Siemens Somatom volume zoom plus 4 .

\section{FNAC procedure \\ Patient positioning and instructions}

Supine/ prone with arms at their sides was chosen generally for maximal patient comfort. Decubitus position was chosen only if absolutely needed because the patients drift out of position easily. Posterior approach was favored if no fissure was violated because it is associated with lesser pneumothorax rate (due to bucket handle motion of ribs).The breathing technique were explained to the patient and practiced beforehand. Deep breaths and coughing were avoided during procedure. Thin sections of CT scans were obtained to identify and target the lesion.

Small lesion in favorable location was preferable to a larger lesion in less desirable location. For small peripheral lesion, a near tangential approach was used so that the needle enters the lung at some distance from target and plenty of room is there for adjustment. Favorable locations included pleural based lesions, upper lung zone lesions and relatively peripheral lesion. Small lesions abutting the heart, nodules in superior portion of right middle lobe just under dome shaped minor fissure and small lesions deep in $\mathrm{CP}$ angles were considered not feasible. Aerated lung was avoided as far as possible to prevent pneumothorax.

\section{FNAC technique}

The patient was kept in designated position and scout images were used to identify the lesion. On the chosen slice entry site was marked with metallic marker placed on the skin. The distance from midline to entry site, the depth from skin and angle of insertion of needle was assessed on the CT monitor console. After the skin entry site was chosen it was sterilized with standardized antiseptic solution. Cutaneous and subcutaneous tissue was infiltrated with lignocaine up to a maximum dose of $20 \mathrm{ml}$ of $2 \%$ solution. Stepwise advancement of the needle was done within plane of gantry, to a position, just outside the parietal pleura, directed at the lesion and the slice was rescanned to assess the position of needle. When the needle was advanced or withdrawn, the patient was asked to suspend respiration for 10-15 seconds. With needle within the target the central stylet was then removed and 10cc syringe was attached. Suction was applied while rotating and moving the needle to and fro during suspended respiration. Procedure was terminated on acquisition of an adequate specimen, development of symptomatic pneumothorax, or development of parenchymal hemorrhage with severe coughing or hemoptysis. An on-site evaluation of the aspirated specimen was performed by pathologist experienced in lung cytology. Multiple slides were made after smearing the aspirate on slides. Half of the slides were fixed with 95\% ethyl alcohol and stained with Papanicolaou (pap) stain while rest of the air dried smears was stained with May-Grunwald Stain (MGG) stain. A post procedure CT scan was also done in all patients to rule out pneumothorax. Post procedure the patient was rolled off the procedure table onto a stretcher with the puncture site dependent. Patients were kept in supervised area so that staff could be alerted if they develop shortness of breath, chest pain or other symptoms within first hour. If complications developed, the clinical condition of the patient guided further management. A complication was considered as minor if it was managed conservatively. If a complication required active management 
such as intubation, chest tube insertion, or blood transfusion, it was classified as major.

\section{OBSERVATIONS}

The present study was conducted in the Department of Respiratory Medicine, $\mathrm{Pt}$ B D Sharma Rohtak on thirty two patients of either sex having lung cancer suspected clinically or on Chest X-ray/CT Thorax. Detailed history and clinical examination, relevant laboratory investigations, plain chest X-ray findings and CT Thorax findings were recorded in designated proforma. CT guided FNAC was done in all cases and all the complications and results of FNAC were recorded. Out of the total 32 patients, $26(81.25 \%)$ were male and 6 $(18.75 \%)$ were females. Most of the patients $(79.98 \%)$ were in $41-70$ years age group. (Fig 1) Twenty three $(71.87 \%)$ patients were smokers whereas rest were non-smokers. Dyspnea was the most common symptom $(81.25 \%)$ followed by cough and chest pain/discomfort (78.12\%). On general physical examination, raised jugular venous pressure was the most common sign seen in 4 patients $(12.5 \%)$ due to malignancy causing obstruction of superior vena cava.

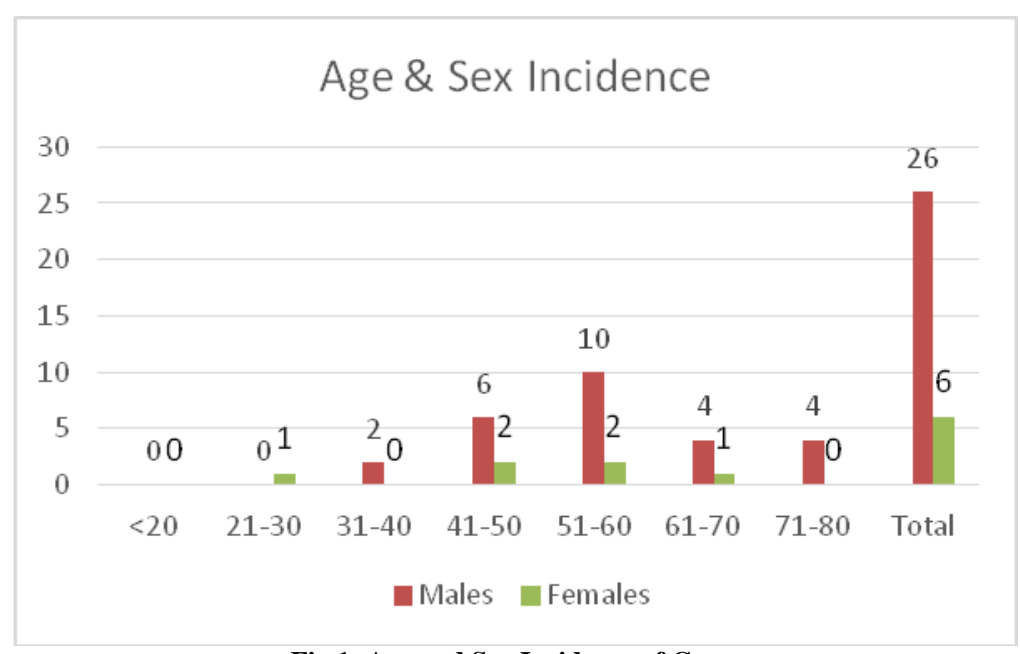

Fig 1: Age and Sex Incidence of Cases

Non enhanced and contrast enhanced CT was performed in all the patients. The mass was located in left upper lobe $(31.25 \%)$ in maximum number of patients. (Table 1)

Table 1 Site of Mass on CT Scan
\begin{tabular}{|l|l|l|}
\hline Site of Mass & Number of Patients & Percentage \\
\hline Mediastinal & 1 & 3.12 \\
\hline Right & 1 & 3.12 \\
\hline Left & 8 & 25 \\
\hline Right Lung field & 2 & 6.25 \\
\hline Right upper lobe & 4 & 12.5 \\
\hline Right Middle Lobe & 3 & 9.37 \\
\hline Right lower Lobe & \multicolumn{1}{|l|}{} \\
\hline Both Lobes & 10 & 31.25 \\
\hline Left Lung Field & 1 & 3.12 \\
\hline Left Upper Lobe & 2 & 6.25 \\
\hline Left lower lobe & \multicolumn{2}{|l|}{} \\
\hline Both Lobes &
\end{tabular}

The lesions were classified based on recommendations by the International Association for Study of Lung Cancer (IASLC) ${ }^{[10]}$ into three sizes i.e. $<3 \mathrm{~cm}, 3$ to
$7 \mathrm{~cm}$ and $>7 \mathrm{~cm} .15$ patients $(46.87 \%)$ had a medium sized mass, 14 patients $(43.75 \%)$ had a large mass and 3 patients $(9.37 \%)$ had a small sized mass. Calcification within mass was seen in 4 patients $(12.5 \%)$.

Pleural effusion and Bony cage erosion was present in 3 patients $(9.37 \%)$ each. In one patient $(3.12 \%)$, vertebral erosion was present. 23 patients $(71.87 \%)$ had mediastinal/ hilar lymphadenopathy. 12 patients $(37.5 \%)$ had liver metastasis, 1 patient $(3.12 \%)$ had an adrenal metastasis and 4 patients $(12.5 \%)$ had both liver and adrenal metastasis. 3 patients $(9.37 \%)$ had pneumothorax as a complication. Chest tube was required in two cases whereas third case resolved spontaneously.

\section{Cytologic Diagnosis on FNAC}

Two patients $(6.25 \%)$ had small cell carcinoma whereas 26 patients $(65.62 \%)$ 
Vipul Kumar et.al. Yield of computed tomography guided fine needle aspiration in diagnosis of suspected cases of lung cancer: experience of a tertiary care hospital of North India.

had non-small cell carcinoma. Out of these 26 patients, 14 had squamous cell carcinoma, 9 had adenocarcinoma and 3 had large cell carcinoma (Figure 2, 3, 4, 5, 6). One patient was found to have small round cell tumor while 3 patients $(9.37 \%)$ had non neoplastic conditions which were inflammatory in nature. Out of 29 diagnosed cases of malignancy, $23(79.31 \%)$ were males and 20(68.96\%) were smokers.

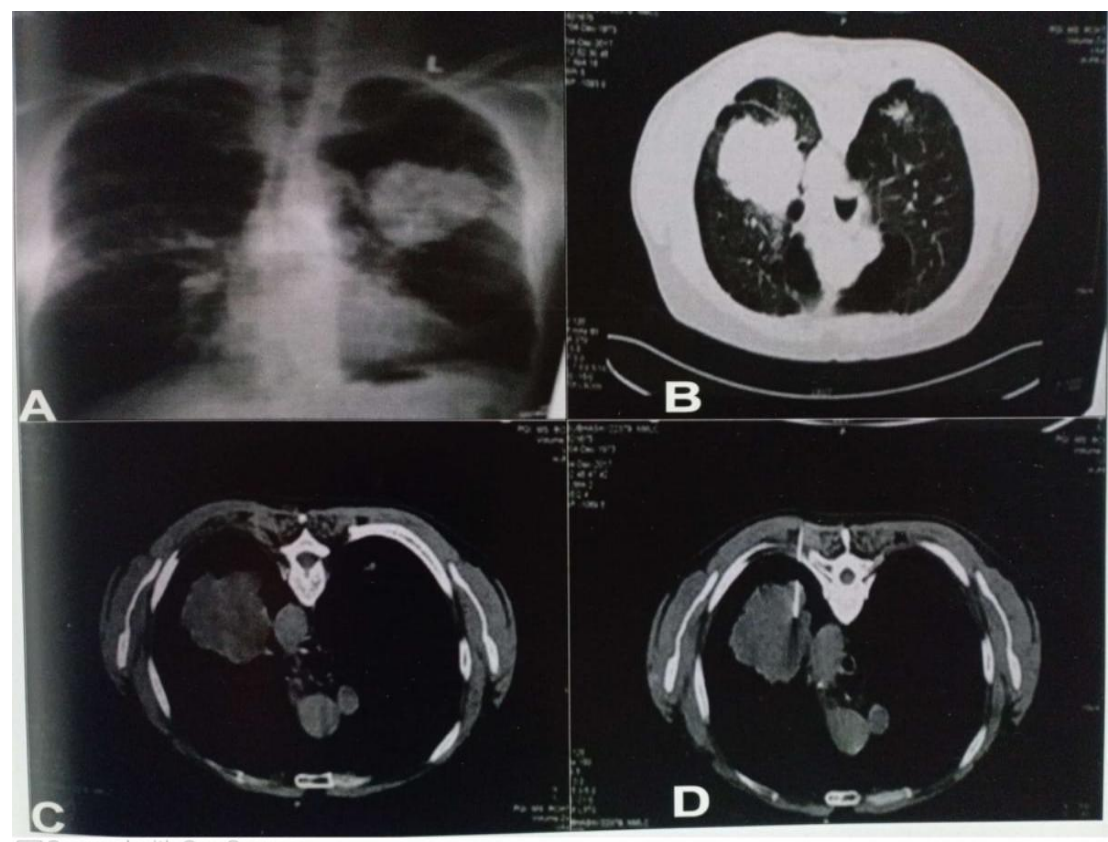

Figure 2: 44 year old male smoker with mass in left upper lobe. CT guided FNAC revealed Squamous Cell Carcinoma

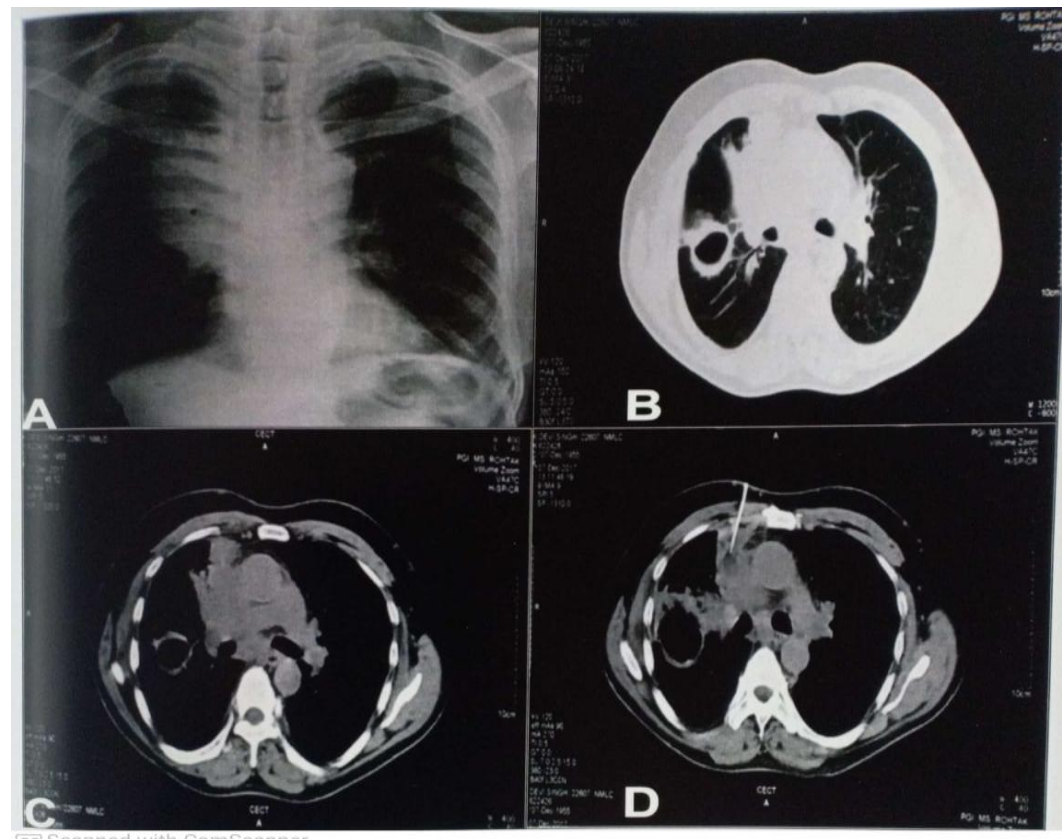

Figure 3: 62 year old male smoker with mass in right hilar region. CT guided FNAC revealed Adenocarcinoma. Image D shows FNAC needle in mass.

Table 2 Cytologic Diagnosis on FNAC

\begin{tabular}{|l|l|l|l|l|l|}
\hline Cytological Diagnosis & No of cases (N=32) & Male & Female & Smoker & Non-smoker \\
\hline Squamous cell carcinoma & $14(43.75 \%)$ & 14 & - & 12 & 2 \\
\hline Adenocarcinoma & $9(28.12 \%)$ & 6 & 3 & 6 & 3 \\
\hline Large cell carcinoma & $3(9.37 \%)$ & 1 & 2 & 1 & 2 \\
\hline Small cell carcinoma & $2(6.25 \%)$ & 2 & - & 1 & 1 \\
\hline Round cell tumor & $1(3.12 \%)$ & - & 1 & - & 1 \\
\hline Inflammatory & $3(9.37 \%)$ & 3 & - & 3 & - \\
\hline
\end{tabular}


Vipul Kumar et.al. Yield of computed tomography guided fine needle aspiration in diagnosis of suspected cases of lung cancer: experience of a tertiary care hospital of North India.

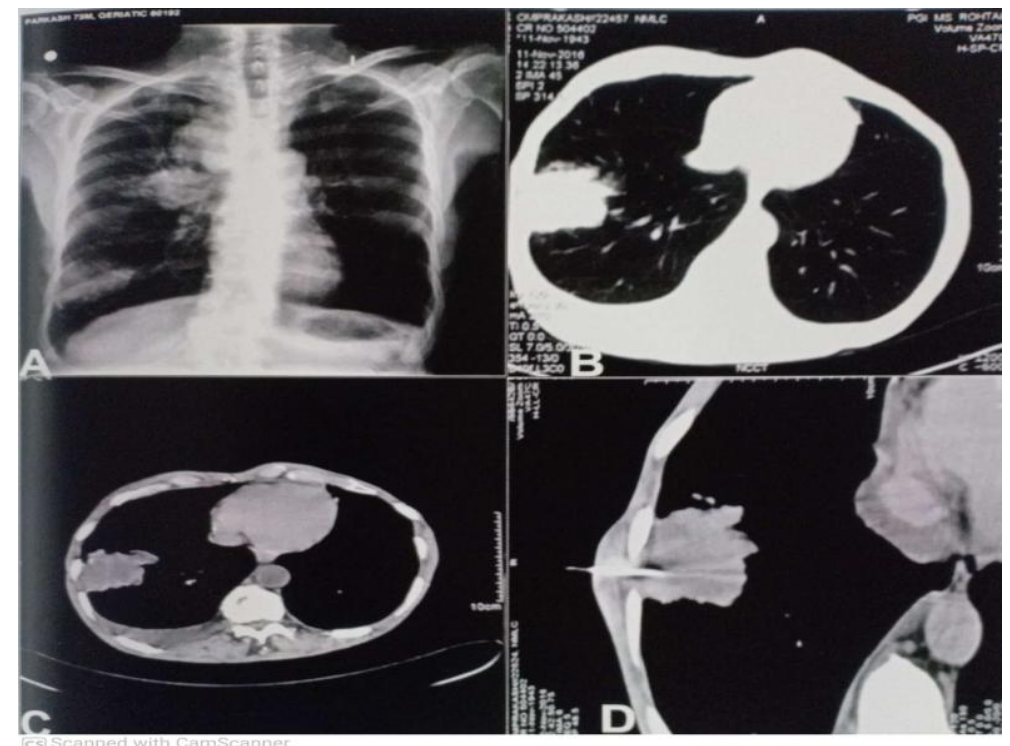

Figure 4: 79 year old male with radio opacity in right lower lobe. CT guided FNAC revealed Small Cell Carcinoma.

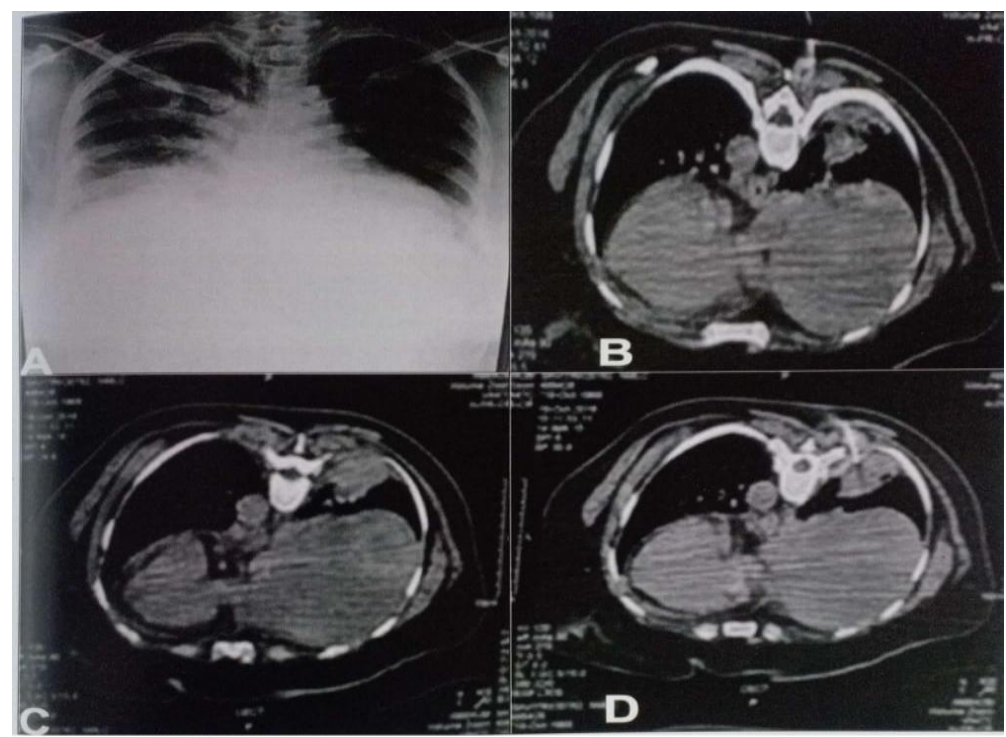

Figure 5: 48 year old female with mass in right lower lobe. CT guided FNAC revealed Large Cell Carcinoma.

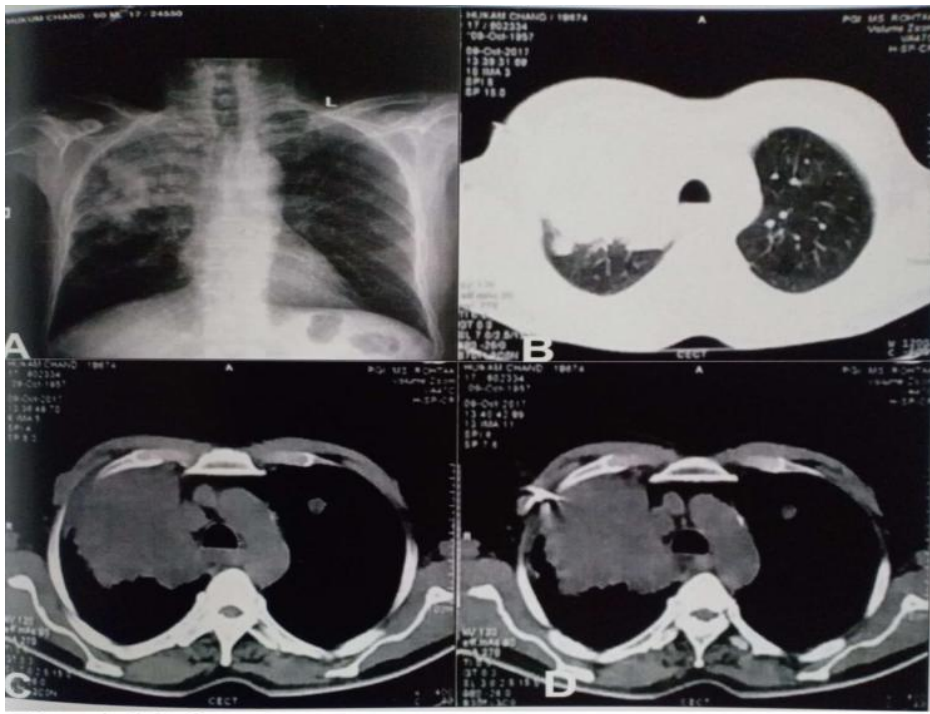

Figure 6: 60 year old male smoker with mass in right upper lobe. CT guided FNAC revealed Squamous Cell Carcinoma. Image D shows FNAC needle in mass.

Galore International Journal of Health Sciences and Research (www.gijhsr.com) 


\section{DISCUSSION}

Modern medical therapy requires precise and specific diagnosis on the nature of a mass. In primary carcinoma of the lung, typing of the neoplasm is important in determining prognosis and in conjugation with the radiographic and clinical manifestations of the neoplasm may influence the decision to operate. When a pulmonary lesion develops in a patient known to have an extra-pulmonary primary neoplasm, it is necessary to determine whether the pulmonary lesion is a metastasis, a second primary neoplasm or a benign lesion. The ultimate aim of any image guided histological or cytological study is to avoid unnecessary thoracotomy and achieve a definite diagnosis with reasonable accuracy and minimum side effects. ${ }^{[11]}$

\section{CT-guided}

percutaneous transthoracic needle aspiration is a wellestablished, effective, and safe technique for the diagnosis of focal lung lesions. ${ }^{[12,13]}$ FNAC provides high-quality material for the microscopic diagnosis of malignancy with high specificity especially when a cytopathologist is present on-site. ${ }^{[14}$

Various factors influence the diagnostic yield like tumor size, location, thickness of the needle, presence of emphysema and the angle of insertion of the needle. Complication and accuracy are influenced by patient factors (age, sex, lung function, and presence of emphysema), lesion variables (size, depth, location, and pleural contact), and procedure-related factors (experience of the operator, degree of difficulty, imaging method used for guidance, and type of needle used). ${ }^{[1]}$

The present study was undertaken to evaluate the usefulness of CT guided FNAC in chest mass lesions. All the patients in the present study were adults and most of the patients $(79.98 \%)$ were in 41-70 years age group. The peak age incidence was 51-60 years that is same as documented by various other studies by Mondal et al ${ }^{[15]}$, Baby and George ${ }^{[16]}$ and Shah et al. ${ }^{[17]}$ The relatively higher cancer rate in elderly population likely reflects the effects of prior smoking.

Male patients showed $(81.25 \%)$ showed significant preponderance in our study compared to females $(18.75 \%)$. In a study by Madan et al, among FNAC-proven cases of malignancy, twenty were males $(83.33 \%)$ and only four were females $(16.66 \%)$ and there was therefore a significant male preponderance with a $\mathrm{M}: \mathrm{F}$ ratio of $5: 1 .^{[18]}$ The reason for higher incidence amongst males may be due to higher smoking prevalence in men.

In our study, lung tumors were located more on the right side $(59.37 \%)$ as compared to the left (40.62\%). Ningthoujam et al ${ }^{[19]}$ found more cases on right side $(55.7 \%)$ than on left side $(44.3 \%)$ and Shanker et al ${ }^{[20]}$ also reported similar findings. Upper lobe was the most common site $(59.37 \%)$ for the lesion among all the patients. Similar findings have been reported by Ningthoujamet al ${ }^{[19]}$ and Saha et al. ${ }^{[21]}$

Most of the patients $(90.62 \%)$ in our study had medium to large sized mass. In patients with lesions $5-10 \mathrm{~mm}$ in size, the prevalence of lung cancer ranges from $6 \%$ to $28 \%$ and for nodules over $2 \mathrm{~cm}$ it varies from $64 \%$ to $82 \%$. Morphological characterization is necessary in all such patients. ${ }^{[22]}$ In a study by Sengupta et al, ${ }^{[23]}$ all the malignant lesions were larger than 2 $\mathrm{cm}$ in diameter. Nodule size is closely correlated to risk of malignancy. Several series have demonstrated an increased incidence of malignancy with increasing nodule size. Nodules larger than $3 \mathrm{~cm}$ will be malignant $80 \%$ to $99 \%$ of the time. In different studies evaluating nodules detected in lung cancer screening trials, the prevalence of malignancy was $0 \%$ to $1 \%$ in patients with nodules $<5 \mathrm{~mm}$ in diameter, $6 \%$ to $28 \%$ for 5 - to $10-\mathrm{mm}$ nodules, $33 \%$ to $64 \%$ for $11-$ to $20-\mathrm{mm}$ nodules and $64 \%$ to $82 \%$ for nodules measuring $>20 \mathrm{~mm}$. $[22,24,25]$ It is now recognized that lesions larger than $3 \mathrm{~cm}$ are almost always malignant, so current convention is that solitary pulmonary nodules must be $3 \mathrm{~cm}$ or 
less in diameter. Larger lesions should be referred to as pulmonary masses and should be managed with the understanding that they are most likely malignant; prompt diagnosis and resection is usually advisable. [26]

We noticed pneumothorax as a complication in 3 patients leading to complication rate of $9.37 \%$. Chest tube was required in two cases whereas third case resolved spontaneously. In a recent crosssectional analysis of 15,865 patients, the rate of any pneumothorax was $15 \%$ and the rate of pneumothorax requiring a chest tube was $7 \% .{ }^{[27]}$ Mohammad et al ${ }^{[28]}$, Gupta et al $^{[29]}$, Baby et al ${ }^{[16]}$ reported pneumothorax in $1.1 \%, 2.7 \%$ and $3.14 \%$ cases respectively.

Table 3: Diagnostic Accuracy and Complication Rates among Different Studies

\begin{tabular}{|c|c|c|c|c|c|}
\hline Study Name (year) & No of cases & Diagnostic Accuracy & Pneumothorax & Hemorrhage & Hemoptysis \\
\hline Stanley et $\mathrm{al}^{[30]}(1987)$ & 458 & $96.6 \%$ & $133(29 \%)$ & - & $5(1.1 \%)$ \\
\hline Vansonnenberg et al ${ }^{[31]}$ (1988) & 150 & $82.7 \%$ & $64(42.7 \%)$ & $2(1.3 \%)$ & $5(3.3 \%)$ \\
\hline Haramati et al ${ }^{[32]}(1995)$ & 32 & $81 \%$ & $3(9.4 \%)$ & - & - \\
\hline Santambrogio etal $^{[33]}$ (1997) & 110 & $81 \%$ & $23(20.9 \%)$ & - & - \\
\hline Gouliamos et al $^{[34]}(2000)$ & 64 & $98.4 \%$ & $2(3.1 \%)$ & $1(1.5 \%)$ & - \\
\hline 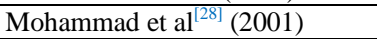 & 184 & $97 \%$ & $2(1.1 \%)$ & - & $10(5.4 \%)$ \\
\hline Gupta et al ${ }^{[29]}(2002)$ & 37 & $91 \%$ & $1(2.7 \%)$ & $1(2.7 \%)$ & - \\
\hline JP Singh et al ${ }^{[6]}(2004)$ & 34 & $85.3 \%$ & $4(11.8 \%)$ & $4(11.8 \%)$ & $1(2.9 \%)$ \\
\hline Baby and George et al ${ }^{[16]}(2014)$ & 114 & $87.7 \%$ & $3(3.14 \%)$ & - & $2(2.28 \%)$ \\
\hline Present Series & 32 & $90.6 \%$ & $3(9.37 \%)$ & - & - \\
\hline
\end{tabular}

In our study, out of total 32 cases, 3 (9.37\%) cases were benign and $29(90.62 \%)$ cases were malignant. Most of the studies have diagnostic accuracy in the range of $80 \%$ to $98 \%$ as is evident from Table 2. However, Basnet et $\mathrm{al}^{[35]}$ reported only $62 \%$ accuracy in their study. We found that 2 patients $(6.25 \%)$ had small cell carcinoma whereas 26 patients $(65.62 \%)$ had non-small cell carcinoma. Out of these 26 patients, $14(43.75 \%)$ had squamous cell carcinoma, 9(28.12\%) had adenocarcinoma and $3(9.37 \%)$ had large cell carcinoma. One patient was found to have small round cell tumor while 3 patients $(9.37 \%)$ had non neoplastic conditions which were inflammatory in nature. Squamous cell carcinoma was the most common type of malignancy detected followed by adenocarcinoma. These findings were consistent with other studies done by Basnet et al ${ }^{[35]}$, Gupta and Mrigpuri ${ }^{[36]}$, Madan and Bannur ${ }^{[18]}$ and Jayashanker et al [20] showing that squamous cell carcinoma is the most common type of malignancy in this part of the country. In contrast to our findings, Mondal et al [15] and Gangopadhyay et al [9] reported adenocarcinoma to be most common type of malignancy comprising $51.72 \%$ and $52.4 \%$ of cases. Singh et al ${ }^{[6]}$ found equal number cases for both squamous cell carcinoma and adenocarcinoma. We found small cell carcinoma in 2 patients $(6.25 \%)$ while Parajuliet al ${ }^{[37]}$, Mondal etal ${ }^{[15]}$ and Jayashanker et al ${ }^{[20]}$ detected small cell carcinoma in $14.9 \%, 6.89 \%$ and $3 \%$ cases respectively.

In the recent past, a relative increase in the incidence of adenocarcinoma has been witnessed. In most of developed countries, it has become the dominant histological type of lung cancer. This histological shift (increase in the incidence of adenocarcinoma) in the developed countries has been linked to changes on the smoking behavior of the population in these regions as well as in the method of manufacturing and composition of cigarettes being marketed therein. Use of cigarettes that had filters as well as lower content of tar and nicotine resulted in a tendency for their smoke to be inhaled more deeply. This led to a higher incidence of carcinogens in the peripheral areas of the lungs where adenocarcinoma tends to form. One of the likely reasons for the lack of change in the epidemiology of lung cancer in India and possibly other developing countries is the fact that the most common smoking product is not cigarette, but bidi - the hand rolled form of tobacco rolled in the dried tendu 
leaf. The process of bidi manufacturing is primarily a cottage industry and has changed little with time unlike that for cigarettes. Therefore an absence of change in the smoking pattern of the Indian population may be another reason for the lack of change in the epidemiology of lung cancer.

\section{CONCLUSION}

CT guided FNAC is a simple, safe, and reliable procedure with high diagnostic accuracy for the diagnosis and sub typing of lung cancer. This helps in early diagnosis and sub-classification of malignant lesions in developing countries having extreme disease burden and resource shortage thereby avoiding more costly and invasive surgical procedures. Though complications are rare, pneumothorax and hemoptysis are occasionally encountered but resolve with active management.

\section{Acknowledgement: None}

\section{Conflict of Interest: None}

\section{Source of Funding: None}

\section{Ethical Approval: Approved}

\section{REFERENCES}

1. Surveillance, epidemiology and end results (SEER) statistics fact sheets: Lung and Bronchus by National Cancer Institute. Available from: http://www.seer.cancer.gov/statfacts/html/ lungb.html (accessed on 9/9/2016)

2. Boiselle PM, Patz EF, Vining DJ, Weissleder R, Shepard JA, McLound TC. Imaging of mediastinal lymphnodes: CT, MR and FDGPET. Radiographics 1998;18:1061-69

3. Reck M, von Pawel J, Zatloukal P, Ramlau R, Gorbounova V, Hirsh V et al. Phase III trial of cisplatin plus gemcitabine with either placebo or bevacizumab as first-line therapy for nonsquamous non-small-cell lung cancer. J ClinOncol2009; 27: 1227-34
4. Tsao MS, Sakurada A, Cutz JC, Zhu CQ, Kamel-Reid S, Squire Jet al..Erlotinib in lung cancer- Molecular and clinical predictors of outcome. N Engl J Med 2005; 353: 133-44.

5. Sharma RK, Chhabra G, Luhadia A, Sharma S, Luhadia SK. Comparative study of computed tomography guided fine needle aspiration cytology and trucut biopsy in diagnosis of lung cancer: A report of 81 cases. Int $\mathbf{J}$ Res Med Sci 2016; 4: 806-08.

6. Singh JP, Garg L, Setia V. Computed Tomograhy $(\mathrm{CT})$ guided transthoracic needle aspiration cytology in Thoracic mass lesions - not approachable by USG. Ind J Radiol Imag 2004; 395-400.

7. Martin HE,Ellis EB. Biopsy by needle puncture and aspiration. Ann Surg. 1930 Aug ; 92 (2) :169-81.

8. Geraghty PR, Kee ST, Mc Farlane G, Razavi MK, Sze DY, Dake MD. CT guided transthoracic needle aspiration biopsy of pulmonary nodules: needle size and pneumothorax rate. Radiology2003 Nov;229(2):475-81.

9. Gangopadhyay M, Chakrabarti I, Ghosh N,Giri A. Computed tomography guided fine needle aspiration cytology of mass lesions of lung: Our experience. Indian J Med PaediatrOncol 2011; 32(4): 192-96.

10. Wallace MJ, Krishnamurthy S, Broemeling LD, Gupta S, Ahrar K, Morello FA Jr, et al. CT-guided percutaneous fine needle aspiration biopsy of small pulmonary lesions. Radiology 2002;225:823-28.

11. Prashant, Ramachandra C, Pattbhiraman, Raghuram, Attili VSS. Feasibility, safety, and efficacy of the CT guided fine needle aspiration cytology (FNAC) of lung lesions. Indian $\mathrm{J}$ Med Paediatr Oncol 2007; 28: 16-25.

12. Laurent F, Latrabe V, Vergier B, Mountadon M, Mernejoux JM, Dubrez J. CT-guided transthoracic needle biopsy of pulmonary nodules smaller than $20 \mathrm{~mm}$ : results with an automated 20-gauge coaxial cutting needle. ClinRadiol 2000; 55:281-87.

13. Austin JHM, Cohen MB. Value of having a cytopathologist present during 
percutaneous fine-needle aspiration biopsy of lung: report of 55 cancer patients and meta-analysis of literature. AJR Am J Roentgenol 1993; 160: 175-77.

14. Miller DA, Carrasco CH, Katz RL, Cramer FM, Wallace S, Charnsangavej C. Fine needle aspiration biopsy: the role of immediate cytologic assessment. AJR Am J Roentgenol1986; 147: 155-58.

15. Mondal SK, Nag D, Das R, Mandal PK, Biswas PK, Osta M. Computed tomogram guided fine needle aspiration cytology of lung mass with histological correlation: A study in Eastern India. South Asian Journal of Cancer 2013; 2: 14-18.

16. Baby J, George P. Computed tomography guided fine needle aspiration cytology of thoracic lesions: A retrospective analysis of 114 cases. Journal of Dental and Medical Sciences 2014; 13: 47-52.

17. Shah S, Shukla K, Patel P. Role of fine needle aspiration cytology in diagnosis of lung tumors - A study of 100 cases. Indian J Pathol Microbiol 2007;50:56-58.

18. Madan $M$ and Bannur H. Evaluation of Fine Needle Aspiration Cytology in the Diagnosis of Lung Lesions. Turkish Journal of Pathology 2010; 26(1): 1-6

19. Ningthoujam BS, Gurumayum LS, Thokchom DS, Kshetrimayum BS, Konjengbam R, Singh R, et al. Computed Tomography-Guided Fine Needle Aspiration Cytology of intrathoracic mass lesions - A two years cross sectional study of 76 cases. Journal of Dental and Medical Sciences 2015; 14(9): 1-8

20. Jaya Shankar E., Pavani B., Chandra E., Reddy R., Srinivas M., Shah A. CT Guided Percutaneous Thoracic FNAC in Lung and Mediastinum, J Cytol Histol 2010; 1(3): 107-10.

21. Saha A, Kumar K, Choudhuri MK. Computed tomography-guided fine needle aspiration cytology of thoracic mass lesions: A study of 57 cases. Journal of Cytology 2009; 26(2): 55-57.

22. Wahidi MM, Govert JA, Goudar RK, Gould MK, McCrory DC, American College of Chest Physicians. Evidence for the treatment of patients with pulmonary nodules: When is it lung cancer?: ACCP evidence-based clinical practice guidelines $\left(2^{\text {nd }}\right.$ edition). Chest $2007 ; 132$ : 94S-107.

23. Sengupta M, Saha K. Computed tomography guided fine needle aspiration cytology of pulmonary mass lesions in a tertiary care hospital: A two-year prospective study. Med J DY Patil Univ 2014; 7: 177-81.

24. Trunk G, Gracey DR, Byrd RB. The management and evaluation of the solitary pulmonary nodule. Chest. 1974;66(3): 236-39.

25. Libby DM, Henschke CI, Yankelevitz DF. The solitary pulmonary nodule: update 1995. Am J Med. 1995;99(5):491-96.

26. Almeida FA, Uzbeck M, Ost D. Initial evaluation of the non-small cell lung cancer patient: diagnosis and staging. Curr Opin Pulm Med. 2010;16(4):307-14.

27. Wiener RS, Schwartz LM, Woloshin S, Welch HG. Population based risk for complications after transthoracic needle lung biopsy of a pulmonary nodule: an analysis of discharge records. Ann Intern Med. 2011;155(3):137-44.

28. Mohammad GM. CT guided fine needle aspiration cytology in the diagnosis of thoracic lesions. JIMA 2001; 99(10): 1-5

29. Gupta S, Michael JW, Frank AM, Kamran A, Marshall EH. CT guided percutaneous needle biopsy of intrathoracic lesions by using the trans-sternal approach: experience in 37 patients. Radiology 2002; 222: 57-62

30. Stanley JH, Fish GD, Andriole JG, Gobien RP, Betsill WL, Laden SA, et al. Lung lesions: cytological diagnosis by fine needle biopsy. Radiology 1987; 162: 389-91

31. Vansonnenberg E, Casola G, Ho M, Neff CC, Varney RR, Wittich GR, et al. Difficult thoracic lesions: CT-guided biopsy experience in 150 cases. Radiology. 1988; 167(2): 457-61.

32. Haramati LB. CT-guided automated needle biopsy of the chest. AJR 1995; 165: 53-55.

33. Santambrogio L, Nosotti M, Bellaviti N, Pavoni G, Radice F, Caputo V. CTguided fine-needle aspiration cytology of solitary pulmonary nodules: a prospective, 
Vipul Kumar et.al. Yield of computed tomography guided fine needle aspiration in diagnosis of suspected cases of lung cancer: experience of a tertiary care hospital of North India.

randomized study of immediate cytologic evaluation. Chest. 1997; 112(2): 423-25.

34. Gouliamos AD, Giannopoulos DH, Panagi GM, Fletoridis NK, Deligeorgi-Politi HA, Vlahos LJ. Computed tomography-guided fine needle aspiration of peripheral lung opacities: An initial diagnostic procedure Actacytologica 2000; 44 (3): 344-48.

35. Basnet SB, Thapa GB, Shahi R, Shrestha M, Panth R. Computed Tomography Guided Percutaneous Transthoracic Fine Needle Aspiration Cytology in Chest Masses. J Nepal Med Assoc 2008; 47(171): 123-7

36. Gupta A, Mrigpuri P. Assessment of clinico-radiological correlation with $\mathrm{CT}$ guided FNAC of different lung lesions: a hospital based study. International Journal of Contemporary Medical Research 2017; 4(6):1290-93.

37. Parajuli S, Tuladhar A, Basnet RB. Ultrasound and computed tomography guided fine needle aspiration cytology in diagnosing intra-abdominal and intrathoracic lesions. J Pathol Nepal 2011;1: 17-21.

How to cite this article: Kumar V, Yadav R, Verma $S$ et.al. Yield of computed tomography guided fine needle aspiration in diagnosis of suspected cases of lung cancer: experience of a tertiary care hospital of North India. Gal Int J Health Sci Res. 2021; 6(3): 46-56. DOI: https://doi.org/10.52403/gijhsr.20210708 